\title{
Effects of Fasting and Refeeding on Expression of Proteolytic- Related Genes in Skeletal Muscle of Chicks
}

\author{
Kazuki NAKASHIMA, Tokushi Komatsu, Makoto YAMAZAKI and Hiroyuki ABE \\ Department of Animal Physiology and Nutrition, National Institute of Livestock and Grassland Science, \\ Tsukuba, Ibaraki 305-0901, Japan \\ (Received January 17, 2005)
}

\begin{abstract}
Summary This experiment was conducted to study the effects of fasting and refeeding on proteolytic-related gene expression in skeletal muscles of chicks. Chicks were fasted for $24 \mathrm{~h}$, and refed for $2 \mathrm{~h}$. Plasma $N^{\tau}$-methylhistidine concentration, as an index of myofibrillar protein degradation, was increased by fasting, and that increment was reduced by refeeding. We also examined the expression of the protease mRNAs (calpain, proteasome, cathepsin and caspase-3) by real-time PCR of cDNA in skeletal muscles of fasting and refeeding chicks. Calpain (m-, $\mu$-, and p94/calpain-3) mRNA expressions were also increased by fasting, and their increment was reduced by refeeding. Ubiquitin and $20 S$ proteasome $\alpha$ subunit ( $\alpha 6$ and $\alpha 7)$ mRNA expressions as well as cathepsin B, and caspase-3 mRNA expression were likewise increased by fasting, with their increment also reduced by refeeding. These results indicate that fasting stimulates proteolytic-related gene expression, resulting in an increase in myofibrillar protein degradation, and that refeeding suppresses proteolytic-related gene expression, resulting in a decrease in myofibrillar protein degradation in chicks.
\end{abstract}

Key Words fasting, refeeding, myofibrillar proteolysis, gene expression, skeletal muscle

Fasting induces an increase in the protein degradation of skeletal muscle in humans and laboratory animals (1-7). On the other hand, feeding stimulates anabolism through a suppression of proteolysis and a stimulation of protein synthesis (8), although the precise mechanism responsible for these effects remains to be elucidated. Much is known about the effects of longterm changes in dietary intake on protein metabolism, but less is known about the acute response to feeding. Rates of protein synthesis in tissues of growing animals are highly sensitive to the immediate intake of food (9), and muscle protein synthesis in animals is particularly sensitive to food intake $(9,10)$; however, it is not clear whether changes in muscle protein degradation occur in vivo in response to food intake. Although our knowledge of the cellular and molecular mechanisms of proteolysis has increased dramatically in recent years (11), there is very little information about the in vivo nutritional regulation of the different proteolytic pathways in tissues (12).

Like other tissues, skeletal muscle contains multiple proteolytic systems, each of which could play an important role in muscle atrophy. Intracellular proteolysis is carried out by lysosomal and non-lysosomal pathways in which intracellular proteases are directly responsible for the degradation of proteins. Calpains, i.e., cysteine proteases in the cytosol, are thought to be the main agents of non-lysosomal $\mathrm{Ca}^{2+}$-dependent proteolysis which occurs within the myofibril, and have been shown capable of carrying out the initial step in myo-

E-mail: kaznaka@affrc.go.jp fibrillar proteolysis $(13,14)$. Proteasome, the multicatalytic proteinase complexes in the cytosol, is also thought to be responsible for non-lysosomal ATPdependent proteolysis (15). Although proteasome has been implicated in the regulation of myofibrillar protein degradation $(16,17)$, its substrates and the control of its activity have not been fully studied in muscles. Proteasome may degrade muscle proteins released due to the action of the other proteases. Cathepsins, i.e., the main agents of lysosomal degradation, have been well established as contributing to muscle protein breakdown (18). Lysosomal proteases degrade sarcoplasmic proteins and released myofibrillar proteins (19). Recently, caspase-3, an apoptotic protease, has been shown to be an initial step triggering accelerated muscle proteolysis in catabolic conditions (20). However, the precise roles of all these degradation systems in the breakdown of skeletal muscle proteins have yet to be determined.

To clarify these issues, the present study investigated the effects of fasting and refeeding on proteolyticrelated gene expression in skeletal muscles of chicks.

The degradation rate of skeletal muscle protein can be measured by the urinary excretion of $N^{\tau}$-methylhistidine, which is localized in the myofibrillar proteins, myosin and actin, but it is not reused for protein synthesis (21). Measuring the urinary excretion of $N^{\tau}$ methylhistidine will not detect acute changes in myofibrillar protein degradation, since the collection period for urine is usually $1 \mathrm{~d}$. In contrast, the plasma $N^{\tau}$ methylhistidine concentration can reflect an acute change in the degradation of myofibrillar protein $(7$, 22). 
Table 1. Gene sequences used as forward and reverse primers for real-time PCR.

\begin{tabular}{|c|c|c|c|}
\hline \multirow{3}{*}{$\begin{array}{r}\text { Gene } \\
\text { Ubiquitin }\end{array}$} & \multicolumn{2}{|c|}{ Sequence $5^{\prime}-3^{\prime}$} & \multirow{2}{*}{$\begin{array}{c}\text { Amplicon (bp) } \\
152\end{array}$} \\
\hline & Forward primer & CGC ACC CTG TCT GAC TAC AA & \\
\hline & Reverse primer & GCC TTC ACG TTC TCA ATG GT & \\
\hline \multirow[t]{2}{*}{$\alpha 6$} & Forward primer & AAC ACA CGC TGT TCT GGT TG & 241 \\
\hline & Reverse primer & CTG CGT TGG TAT CTG GGT TT & \\
\hline \multirow[t]{2}{*}{$\alpha 7$} & Forward primer & ACG GTG TAC GAG CTG AGG AA & 164 \\
\hline & Reverse primer & CCT TCG CTG TCC ACG TAG TA & \\
\hline \multirow{2}{*}{ m-Calpain } & Forward primer & ACA TCA TCG TGC CCT CTA CC & 202 \\
\hline & Reverse primer & CTG CGT TGG TAT CTG GGT TT & \\
\hline \multirow[t]{2}{*}{$\mu$-Calpain } & Forward primer & GGG CTA CAA ACT GAC CCA AA & 204 \\
\hline & Reverse primer & TAG GCA AAC ATG GTG AGC TG & \\
\hline \multirow{2}{*}{ p94/calpain-3 } & Forward primer & GAT ATG CCG GGA AGA ACT CA & 223 \\
\hline & Reverse primer & GGT TCC TGA ATG GTC TGC AT & \\
\hline \multirow[t]{2}{*}{ Cathepsin B } & Forward primer & CAA GCT CAA CAC CAC TGG AA & 150 \\
\hline & Reverse primer & TCA AAG GTA TCC GGC AAA TC & \\
\hline \multirow[t]{2}{*}{ Caspase-3 } & Forward primer & TGG CGA TGA AGG ACT CTT CT & 173 \\
\hline & Reverse primer & CTG GTC CAC TGT CTG CTT CA & \\
\hline \multirow[t]{2}{*}{ GAPDH } & Forward primer & CCT CTC TGG CAA AGT CCA AG & 200 \\
\hline & Reverse primer & CAT CTG CCC ATT TGA TGT TG & \\
\hline
\end{tabular}

Last column indicates the length of amplicon (bp). $\alpha 6,20$ s proteasome subunit $\alpha-6 ; \alpha 7,20$ S proteasome $\alpha-7$ subunit; mcalpain, m-calpain large subunit; $\mu$-calpain, $\mu$-calpain large subunit; p94/calpain-3, p94/calpain-3 large subunit.

\section{MATERIALS AND METHODS}

Animal preparation and experimental protocol. Oneday-old male layer chicks were supplied by a local commercial hatchery (Kasumigaura-Furanjo, Ibaraki, Japan). They were housed in an electrically-heated battery brooder and were provided with water and a commercial starter diet (Shimizukou-shiryou, Shizuoka, Japan) ad libitum for $7 \mathrm{~d}$. On day 7, 18 birds of similar body weight (about $80 \mathrm{~g}$ ) were selected and housed in wire-bottomed aluminum cages, and six replications were made per treatment. The temperature of the room was $25^{\circ} \mathrm{C}$, and relative humidity was maintained at 50 $70 \%$ throughout the experiment. They were given free access to a semi-purified corn soybean meal diet (crude protein $20 \%$, metabolizable energy $2,900 \mathrm{kcal} / \mathrm{kg}$ diet) and water for $7 \mathrm{~d}$. At the start of the experiment, 14-dold chicks weighing $140 \pm 3 \mathrm{~g}$ were divided into groups and caged separately. Fed chicks were maintained as described above. Food-deprived chicks had food removed $24 \mathrm{~h}$ before they were killed. Refed chicks were food-deprived for $24 \mathrm{~h}$ and then refed for $2 \mathrm{~h}$. The experimental procedures used in this study met the guidelines of the Animal Care and Use Committee of the National Institute of Livestock and Grassland Science.

RNA isolation and real-time PCR. Gastrocnemius muscles were rapidly excised, frozen in liquid nitrogen and stored at $-80^{\circ} \mathrm{C}$. Total RNA was extracted using ISOGEN Reagent (Nippon Gene Co., Ltd., Tokyo, Japan) according to the manufacturer's protocols. cDNA was synthesized from 1-1,000 ng of total RNA using random hexamer (TaKaRa, Tokyo, Japan) and Murine Moloney leukemia virus reverse transcriptase (Gibco Life Technologies, Gaithersburg, MD, USA). Real-time PCR primers were designed (software Primer3, http:// www.broad.mit.edu/cgi-bin/primer/primer3_www.cgi) for ubiquitin, 20S proteasome subunit $\alpha 6,20 S$ proteasome $\alpha 7$ subunit, m-calpan large subunit, $\mu$-calpain large subunit, p94/calpain-3 large subunit, cathepsin B, caspase-3 and GAPDH (Table 1). The expression of mRNA was measured by real-time PCR using a LightCycler (Roche Diagnostics, Mannheim, Germany) instrument with the QuantiTect SYBR Geen PCR system (Qiagen K.K., Tokyo, Japan) starting with 5 ng of reversetranscribed total RNA. GAPDH expression was used as an internal control. For quantification of the levels of mRNA expression, PCR product roughly equivalent in size and equivalent primer lengths and GC contents of each primer $(50-60 \%)$ were selected. PCR was performed under the following conditions: $95^{\circ} \mathrm{C} \times 15 \mathrm{~min}$, $45 \times\left(94^{\circ} \mathrm{C} \times 15 \mathrm{~s}, 55^{\circ} \mathrm{C} \times 20 \mathrm{~s}, 72^{\circ} \mathrm{C} \times 12 \mathrm{~s}\right)$.

$\mathrm{N}^{\tau}$-methylhistidine analysis. Plasma $N^{\tau}$-methylhistidine concentration was measured by the HPLC method after derivatization of fluorescamine with a treatment of perchloric acid and heating (23).

Statistical analysis. Data were analyzed by one-way analysis of variance and Duncan's multiple comparison test. A $p$ of value $<0.05$ was considered statistically significant. Each result is expressed as the mean \pm SD.

\section{RESULTS AND DISCUSSION}

In the present study, the effect of fasting and refeeding on myofibrillar proteolysis and protease (calpain, proteasome, cathepsin and caspase) mRNA expressions in skeletal muscles of chicks was investigated. The results of body weight, gastrocnemius muscle weight, and plasma $N^{\tau}$-methylhistidine concentration are shown in Table 2. Body weight was significantly decreased by fasting, and refeeding improved growth inhibition. Although gastrocnemius muscle was also 
Table 2. Effects of fasting and refeeding on body weight, gastrocnemius muscle, and plasma $N^{\tau}$-methylhistidine concentration of chicks.

\begin{tabular}{lccc}
\hline & Fed & Fasted & Refed \\
\hline Body weight $(\mathrm{g})$ & $146.8 \pm 5.7^{\mathrm{a}}$ & $125.5 \pm 2.8^{\mathrm{b}}$ & $143.1 \pm 5.2^{\mathrm{a}}$ \\
Gastrocnemius muscle & $0.48 \pm 0.06^{\mathrm{a}}$ & $0.36 \pm 0.04^{\mathrm{b}}$ & $0.37 \pm 0.02^{\mathrm{b}}$ \\
$\quad$ weight $(\mathrm{g})$ & $23.1 \pm 1.6^{\mathrm{b}}$ & $37.3 \pm 3.3^{\mathrm{a}}$ & $25.6 \pm 2.4^{\mathrm{b}}$ \\
$\quad$ Plasma $N^{\tau}$-methylhistidine & & \\
$\quad$ concentration $(\mathrm{nmol} / \mathrm{mL})$ & & \\
\hline
\end{tabular}

Values represent means $\pm \mathrm{SD}, n=6$.

Values with different letters are significantly different $(p<0.05)$.

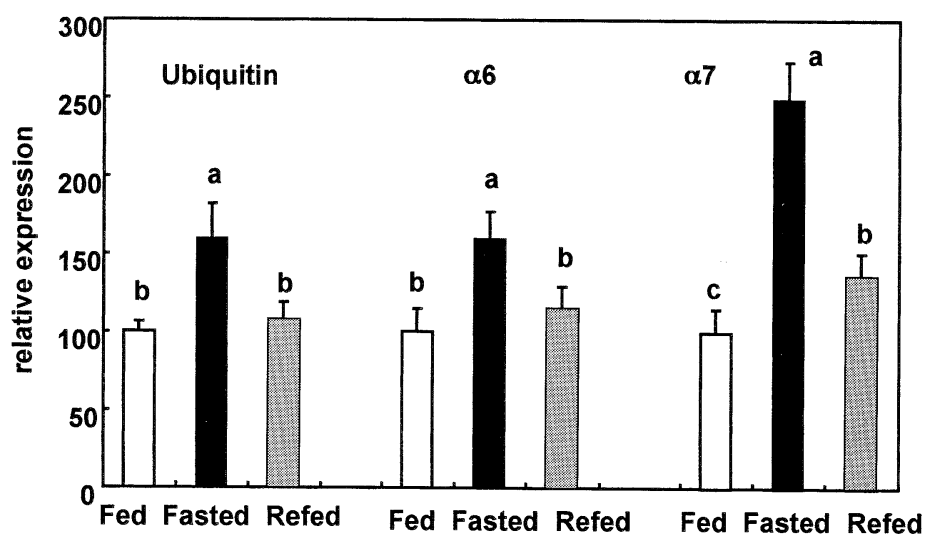

Fig. 1. Quantification of mRNA levels encoding ubiquitin, and 20S proteasome subunits ( $\alpha 6$ and $\alpha 7$ ) in gastrocnemius muscles of fasted and refed chicks. Results are expressed as mRNA relative expression and are means \pm SD for 5-6 animals. RNA quantification was performed as indicated in the Materials and Methods. Values that do not share the same superscript letter are significantly different $(p<0.05)$.

significantly decreased by fasting, refeeding failed to restore muscle weight to the fed level within $2 \mathrm{~h} . N^{\tau_{-}}$ methylhistidine concentration, as an index of myofibrillar proteolysis, was increased by fasting, whereas refeeding suppressed it to the fed level. $N^{\tau}$-methylhistidine is an amino acid formed by the post-translational methylation of specific histidine residues in the myofibrillar proteins, actin and myosin. Since $N^{\tau}$-methylhistidine cannot charge tRNA, it cannot be reutilized for protein synthesis. Because of this, and also because it does not undergo catabolism, the output of $N^{\tau}$-methylhistidine has been used as an index of myofibrillar proteolysis (21). It has been reported that plasma $N^{\tau}$-methylhistidine concentration, urinary $N^{\tau}$-methylhistidine excretion, and $N^{\tau}$-methylhistidine release from incubated muscle were increased by starvation in rats (7). We have also reported that serum deprivation induces myofibrillar proteolysis as an index of $N^{\tau}$-methylhistidine release into the medium in chick myotube cultures (24). Cells in culture show a series of changes in intracellular protein degradation in response to serum deprivation that resembles alterations in the degradation in tissues of starved animals. During starvation, proteolysis in muscle increases and helps provide the organism with essential amino acids for gluconeogenesis (25). It has been reported that both plasma $N^{\tau}$-methylhistidine concentration $(22,26)$ and $N^{\tau}$-methylhistidine release from incubated muscle (2) were inhibited by food intake after food starvation in rats. These results are also consistent with ours.

The main purpose of the present experiments was to determine the proteolytic mechanism(s) involved in the fasting-dependent increase and the refeeding-dependent decrease in myofibrillar proteolysis. The biochemical pathways for the degradation of muscle protein and its mode of activation under fasting and refeeding remain unclear. We examined the expression of the protease mRNAs (calpain, proteasome and cathepsin, caspase) by real-time PCR of cDNA in skeletal muscles of fasting and refeeding chicks. We measured the mRNA levels of components of the ubiquitin-proteasome system (non-lysosomal ATP-dependent proteolysis). The results of the mRNA expression of ubiquitin and $20 S$ proteasome subunits ( $\alpha 6$ and $\alpha 7$ ) are shown in Fig. 1. Ubiquitin mRNA expression was significantly increased by fasting, and refeeding significantly decreased it to the fed level within $2 \mathrm{~h}$. The $20 \mathrm{~S}$ proteasome $\alpha 6$ and $\alpha 7$ subunit mRNA expressions were also significantly increased by fasting, and refeeding significantly decreased them to the fed level within $2 \mathrm{~h}$. The ATP-ubiquitin-proteasome-dependent pathway is activated by fasting as well as by the induction of stress hormones such as glucocorticoids that oppose the anabolic effects of insulin (27). Recently, food intake was also found to inhibit ATP-ubiquitin-proteasome proteolysis in skeletal muscles of rats (28). These results are consis- 


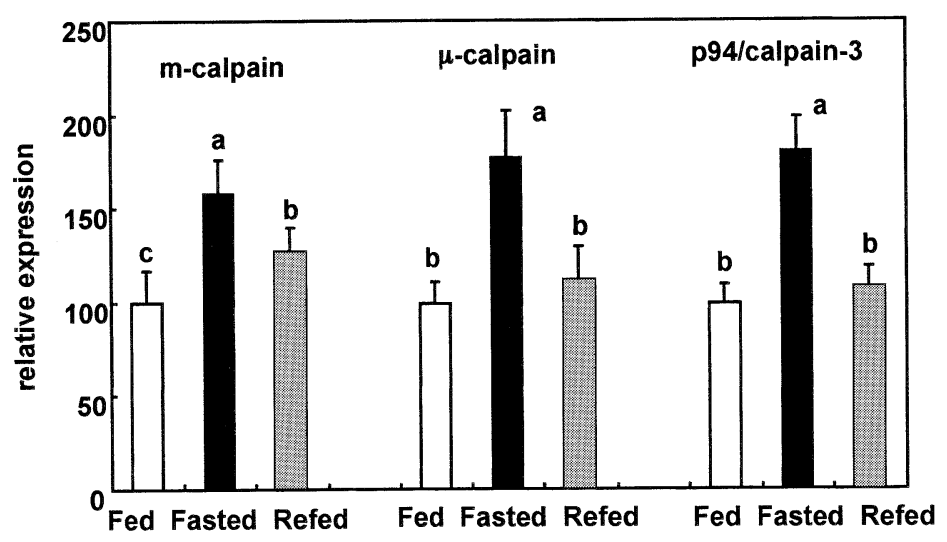

Fig. 2. Quantification of mRNA levels encoding calpain large subunits (m-, $\mu$-, and p94/calpain-3) in gastrocnemius muscles of fasted and refed chicks. Results are expressed as mRNA relative expression and are means \pm SD for 5-6 animals. RNA quantification was performed as indicated in the Materials and Methods. Values that do not share the same superscript letter are significantly different $(p<0.05)$.

tent with ours.

We next measured the mRNA levels for calpains of the non-lysosomal $\mathrm{Ca}^{2+}$-dependent proteolytic system. The results of mRNA expressions of m-calpain, $\mu$ calpain and p94/calpain-3 large subunits are shown in Fig. 2. The $m$-calpain and $\mu$-calpain large subunit mRNA expressions were significantly increased by fasting, and refeeding significantly reduced them to the fed level within $2 \mathrm{~h}$. It has been reported that $\mathrm{m}$ - and $\mu$ calpain mRNA expressions of skeletal muscle were increased by long-term starvation ( 8 d) in rabbits (29). In the present study, we also showed that short-term starvation increased $\mathrm{m}$ - and $\mu$-calpain mRNA expressions in skeletal muscles of chicks. Wing and Goldberg have reported that calpain has not been considered to play an important role in muscle degradation in fasting (27), since it is committed to the limited proteolysis of target proteins (30). They also showed that calpain is not important in muscle proteolysis during fasting by using incubated muscle activates with $\mathrm{Ca}^{2+}$ ionophore A23187-containing medium (27). They did not measure calpain mRNA expression. However, we showed that $\mathrm{m}$ - and $\mu$-calpain expressions were increased by fasting in chicks, indicating calpain has an important role in myofibrillar proteolysis. We have also reported that serum deprivation induces calpain activity in chick myotube cultures. In our model, serum deprivation was shown to be similar to alterations in the degradation of skeletal muscle in starved animals (24). The effect of refeeding on calpain expression in skeletal muscle had not been reported previously. The present results show that refeeding suppresses $\mathrm{m}$ - and $\mu$-calpain expressions in skeletal muscles of chicks. The p94/calpain-3 large subunit mRNA expression was also significantly increased by fasting, and refeeding significantly reduced it to the fed level within $2 \mathrm{~h}$, a finding that had not been reported previously. We showed that short-term fasting increases p94/calpain-3 gene expression and refeeding decreases p94/calpain-3 mRNA expression in skeletal muscles of chicks. The predominant expression of p94/ calpain-3 in skeletal muscle, where mRNA levels are ca.

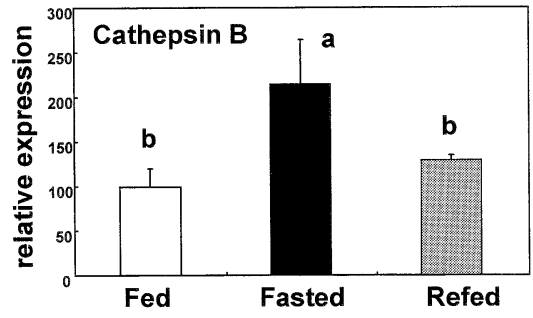

Fig. 3. Quantification of mRNA levels encoding cathep$\sin \mathrm{B}$ in gastrocnemius muscles of fasted and refed chicks. Results are expressed as mRNA relative expression and are means \pm SD for 5-6 animals. RNA quantification was performed as indicated in the Materials and Methods. Values that do not share the same superscript letter are significantly different $(p<0.05)$.

10 times higher than those of $\mathrm{m}$ - and $\mu$-calpain, indicates the physiological importance of p94/calpain-3 in that tissue (31). The muscle-specific calpain, i.e., p94/ calpain-3, has binding sites on connectin/titin, raising the possibility that connectin/titin may be subjected to calcium-dependent proteolysis, thereby disrupting the anchorage of myosin to the Z-disk (32). Hayashi et al. have reported that glucocorticoid (corticosterone) stimulates calpain activity, resulting in a decrease in the connectin/titin content in skeletal muscles of rats (33). The present study provides the first evidence that fasting may be associated with an increased expression of p94/calpain-3.

We next measured the mRNA level for cathepsin B of the lysosomal-dependent proteolytic system. The result of the mRNA expression of cathepsin B is shown in Fig. 3. Cathepsin B mRNA expression was also significantly increased by fasting, and refeeding significantly reduced it to the fed level within $2 \mathrm{~h}$. Muscle lysosomal-dependent proteolysis was also shown to be elevated during fasting (27). Ilian and Forsberg have also reported that rabbit muscle cathepsin D mRNA concentration was increased dramatically by fasting (29). In a previous study, we reported that serum deprivation induces cathepsin B+L and D activities in chick myotube cul- 


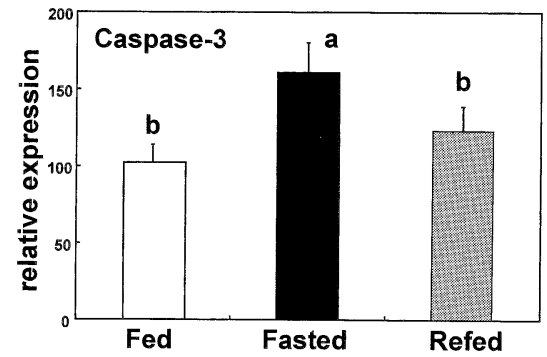

Fig. 4. Quantification of mRNA levels encoding caspase- 3 in gastrocnemius muscles of fasted and refed chicks. Results are expressed as mRNA relative expression and are means \pm SD for 5-6 animals. RNA quantification was performed as indicated in the Materials and Methods. Values that do not share the same superscript letter are significantly different $(p<0.05)$.

tures. In this model, serum deprivation is similar in its effects to alterations in the degradation of skeletal muscle in starved animals (24). On the other hand, food intake is known to inhibit lysosomal proteolysis in liver and skeletal muscle $(4,34)$. However, the effect of refeeding on cathepsin expression in skeletal muscle has not been reported previously. Our present results show that refeeding suppresses cathepsin B expression in skeletal muscles of chicks.

We next measured the mRNA level for caspase-3 of the apoptotic protease. The result of the mRNA expression of caspase-3 is shown in Fig. 4. Caspase-3 mRNA expression was significantly increased by fasting, and refeeding significantly decreased in to the fed level within $2 \mathrm{~h}$, a finding that had not been reported previously. We showed that short-term fasting increases caspase-3 gene expression and refeeding decreases caspase-3 mRNA expression in skeletal muscles of chicks. Recently, caspase- 3 has been shown to be an initial step triggering accelerated muscle proteolysis in diabetes (20). In L6 muscle cells, serum deprivation also induced muscle proteolysis by caspase-3 (20). However, the effect of fasting and refeeding on caspase expression in skeletal muscle has not been reported previously. The present study provides the first evidence that fasting may be associated with an increased expression of caspase-3.

Like other tissues, skeletal muscle contains multiple proteolytic systems, each of which might play an important role in muscle atrophy. Calpains (non-lysosomal $\mathrm{Ca}^{2+}$-dependent proteolysis), proteasome (non-lysosomal ATP-dependent proteolysis), and cathepsins (the main agents of lysosomal degradation) have been well established as contributing to muscle protein breakdown. In the present experiment, we measured calpain, proteasome, cathepsin and caspase expressions, and showed that fasting induces all those expressions in skeletal muscles of chicks.

The present study has shown that the four proteolytic systems found in skeletal muscle are selectively induced fasting and coordinately upregulated in fasting at the molecular level. A specific and transcriptional program that favors muscle atrophy is then likely to be triggered upon food deprivation depending on metabolic and endocrine status. Plasma insulin is widely believed to be a major regulator of the anabolic drive of nutrients. Plasma insulin concentration increases rapidly with refeeding. Insulin reduced muscle protein degradation, through a decrease in the expression of 17 mRNA of ubiquitin ligases and of 11 mRNA of proteasome components in an ATP-ubiquitin-dependent system (35). On the other hand, glucocorticoids are known to be factors which regulate the protein turnover in skeletal muscle. Glucicirticoids stimulate muscle protein degradation, through an increase in the expression of ubiquitin and proteasome subunits in an ATPubiquitin-dependent proteolytic system. It has been demonstrated that the level of ubiquitin and proteasome mRNA were increased with food deprivation $(27$, $28)$ and decreased with refeeding $(27,36)$. In this context, the nature and role of factors such as peripheral hormones regulating proteolytic gene expression still require further investigation.

In conclusion, these results indicate that fasting stimulates protease expressions, resulting in an increase in myofibrillar proteolysis, and refeeding suppresses protease expressions, resulting in a decrease in myofibrillar proteolysis in chicks.

\section{REFERENCES}

1) Wassner SJ, Orloff S, Holliday MA. 1977. Protein degradation in muscle: response to feeding and fasting in growing rats. Am J Physiol 233: E1 19-123.

2) Li JB, Wassner SJ. 1984. Effects of food deprivation and refeeding on total protein and actomyosin degradation. Am J Physiol 246: E32-37.

3) Kadowaki M, Nagasawa T, Hirata T, Noguchi T, Naito H. 1985. Effects of insulin, amino acids and fasting on myofibrillar protein degradation in perfused hindquarters of rats. J Nutr Sci Vitaminol 31: 431-440.

4) Lowell BB, Ruderman NB, Goodman MN. 1986. Regulation of myofibrillar protein degradation in rat skeletal muscle during brief and prolonged starvation. Metabolism 35: 1121-1127.

5) Goodman MN, del Pilar Gomez M. 1987. Decreased myofibrillar proteolysis after refeeding requires dietary protein or amino acids. Am J Physiol 253: E52-58.

6) Hoffer LJ. 1990. Nutritional status affects renal 3-methylhistidine handling in humans. Metabolism 39: 744748 .

7) Nagasawa T, Yoshizawa F, Nishizawa N. 1996. Plasma $N^{\tau}$-methylhistidine concentration is a sensitive index of myofibrillar protein degradation during starvation in rats. Biosci Biotechnol Biochem 60: 501-502.

8) Garlick PJ, McNurlan MA, Bark T, Lang CH, Gelato MC. 1998. Hormonal regulation of protein metabolism in relation to nutrition and disease. J Nutr 128: 356S359S.

9) Yoshizawa F, Kimball SR, Jefferson LS. 1997. Modulation of translation initiation in rat skeletal muscle and liver in response to food intake. Biochem Biophys Res Commun 240: 825-831.

10) Preedy VR, Garlick PJ. 1986. The response of muscle protein synthesis to nutrient intake in postabsorptive rats: the role of insulin and amino acids. Biosci Rep $\mathbf{6}$ : $177-183$. 
11) Attaix D, Combaret L, Pouch MN, Taillandier D. 2001. Regulation of proteolysis. Curr Opin Clin Nutr Metab Care 4: $45-49$.

12) Combaret L, Taillandier D, Attaix D. 2001. Nutritional and hormonal control of protein breakdown. Am J Kidney Dis 37: S108-111.

13) Goll DE, Dayton WR, Singh I, Robson RM. 1991. Studies of the $\alpha$-actinin/actin interaction in the Z-disk by using calpain. J Biol Chem 266: 8501-8510.

14) Goll DE, Thompson VF, Taylor RG, Christiansen JA. 1992. Role of the calpain system in muscle growth. Biochimie 74: 225-237.

15) Coux O, Tanaka K, Goldberg AL. 1996. Structure and functions of the $20 \mathrm{~S}$ and $26 \mathrm{~S}$ proteasomes. Annu Rev Biochem 65: 801-847.

16) Furuno K, Goodman MN, Goldberg AL. 1990. Role of different proteolytic systems in the degradation of muscle proteins during denervation atrophy. J Biol Chem 265: 8550-8557.

17) Solomon V, Goldberg AL. 1996. Importance of the ATPubiquitin-proteasome pathway in the degradation of soluble and myofibrillar proteins in rabbit muscle extracts. J Biol Chem 271: 26690-26697.

18) Hall-Angeras M, Hasselgren PO, Dimlich RV, Fischer JE. 1991. Myofibrillar proteinase, cathepsin B, and protein breakdown rates in skeletal muscle from septic rats. Metabolism 40: 302-306.

19) Lowell BB, Ruderman NB, Goodman MN. 1986. Evidence that lysosomes are not involved in the degradation of myofibrillar proteins in rat skeletal muscle. Biochem J 234: 237-240.

20) Du J, Wang X, Miereles C, Bailey JL, Debigare R, Zheng B, Price SR, Mitch WE. 2004. Activation of caspase-3 is an initial step triggering accelerated muscle proteolysis in catabolic conditions. J Clin Invest 113: 115-123.

21) Young VR, Alexis SD, Baliga BS, Munro HN, Muecke W. 1972. Metabolism of administered 3-methylhistidine. Lack of muscle transfer ribonucleic acid charging and quantitative excretion as 3-methylhistidine and its $\mathrm{N}$ acetyl derivative. J Biol Chem 247: 3592-3600.

22) Nagasawa T, Hirano J, Yoshizawa F, Nishizawa N. 1998. Myofibrillar protein catabolism is rapidly suppressed following protein feeding. Biosci Biotechnol Biochem 62: 1932-1937.

23) Wassner SJ, Schlitzer JL, Li JB. 1980. A rapid, sensitive method for the determination of 3-methylhistidine levels in urine and plasma using high-pressure liquid chromatography. Anal Biochem 104: 284-289.

24) Nakashima K, Nonaka I, Masaki S. 2003. Effects of serum deprivation on myofibrillar proteolysis in chick myotube cultures. Biosci Biotechnol Biochem 67: 2455-
2458.

25) Kettelhut IC, Wing SS, Goldberg AL. 1988. Endocrine regulation of protein breakdown in skeletal muscle. Diabetes Metab Rev 4: 751-772.

26) Yoshizawa F, Nagasawa T, Nishizawa N, Funabiki R. 1997. Protein synthesis and degradation change rapidly in response to food intake in muscle of food-deprived mice. J Nutr 127: 1156-1159.

27) Wing SS, Goldberg AL. 1993. Glucocorticoids activate the ATP-ubiquitin-dependent proteolytic system in skeletal muscle during fasting. Am J Physiol 264: E668676.

28) Kee AJ, Combaret L, Tilignac T, Souweine B, Aurousseau E, Dalle M, Taillandier D, Attaix D. 2003. Ubiquitin-proteasome-dependent muscle proteolysis responds slowly to insulin release and refeeding in starved rats. J Physiol 546: 765-776.

29) Ilian MA, Forsberg NE. 1992. Gene expression of calpains and their specific endogenous inhibitor, calpastatin, in skeletal muscle of fed and fasted rabbits. Biochem J 287: 163-171.

30) Johnson P. 1990. Calpains (intracellular calcium-activated cysteine proteinases): structure-activity relationships and involvement in normal and abnormal cellular metabolism. Int J Biochem 22: 811-822.

31) Sorimachi H, Imajoh-Ohmi S, Emori Y, Kawasaki H, Ohno S, Minami Y, Suzuki K. 1989. Molecular cloning of a novel mammalian calcium-dependent protease distinct from both $\mathrm{m}$ - and $\mu$-types. Specific expression of the mRNA in skeletal muscle. J Biol Chem 264: $20106-$ 20111.

32) Labeit S, Kolmerer B, Linke WA. 1997. The giant protein titin. Emerging roles in physiology and pathophysiology. Circ Res 80: 290-294.

33) Hayashi K, Tada O, Higuchi K, Ohtsuka A. 2000. Effects of corticosterone on connectin content and protein breakdown in rat skeletal muscle. Biosci Biotechnol Biochem 64: 2686-2688.

34) Attaix D, Aurousseau E, Combaret L, Kee A, Larbaud D, Ralliere C, Souweine B, Taillandier D, Tilignac T. 1998. Ubiquitin-proteasome-dependent proteolysis in skeletal muscle. Reprod Nutr Dev 38: 153-165.

35) Rome S, Clement K, Rabasa-Lhoret R, Loizon E, Poitou C, Barsh GS, Riou JP, Laville M, Vidal H. 2003. Microarray profiling of human skeletal muscle reveals that insulin regulates approximately 800 genes during a hyperinsulinemic clamp. J Biol Chem 278: 18063-18068.

36) Medina R, Wing SS, Goldberg AL. 1995. Increase in levels of polyubiquitin and proteasome mRNA in skeletal muscle during starvation and denervation atrophy. Biochem J 307: 631-637. 